\title{
Remote regulation of glucose homeostasis in mice using genetically encoded nanoparticles
}

\author{
Sarah A Stanley ${ }^{\# 1}$, Jeremy Sauer ${ }^{\# 2}$, Ravi S Kane ${ }^{2}$, Jonathan S Dordick ${ }^{2}$, and Jeffrey M \\ Friedman $^{1,3}$ \\ ${ }^{1}$ Laboratory of Molecular Genetics, Rockefeller University, New York, New York, USA \\ ${ }^{2}$ Department of Chemical and Biological Engineering, Department of Biology, Center for \\ Biotechnology and Interdisciplinary Studies, Rensselaer Polytechnic Institute, Troy, New York, \\ USA \\ ${ }^{3}$ Howard Hughes Medical Institute, New York, New York, USA \\ \# These authors contributed equally to this work.
}

\section{Abstract}

Means for temporally regulating gene expression and cellular activity are invaluable for elucidating underlying physiological processes and would have therapeutic implications. Here we report the development of a genetically encoded system for remote regulation of gene expression by low-frequency radio waves (RFs) or a magnetic field. Iron oxide nanoparticles are synthesized intracellularly as a GFP-tagged ferritin heavy and light chain fusion. The ferritin nanoparticles associate with a camelid anti-GFP-transient receptor potential vanilloid 1 fusion protein, aGFPTRPV1, and can transduce noninvasive RF or magnetic fields into channel activation, also showing that TRPV1 can transduce a mechanical stimulus. This, in turn, initiates calciumdependent transgene expression. In mice with stem cell or viral expression of these genetically encoded components, remote stimulation of insulin transgene expression with RF or a magnet lowers blood glucose. This robust, repeatable method for remote regulation in vivo may ultimately have applications in basic science, technology and therapeutics.

Studying the actions of specific cells and gene products in vivo is essential for understanding
their physiological roles. Cellular activity and/or gene expression can be regulated by several
approaches with distinctive features. These range from viruses, which allow constitutive
expression that is quite durable ${ }^{1}$, to rapid photoactivation of caged mRNA ${ }^{2}$, transactivators
or genetically encoded light sensors ${ }^{4}$ that switch on gene expression. However, light delivery

Reprints and permissions information is available online at http://www.nature.com/reprints/index.html.

Correspondence should be addressed to J.M.F. (; Email: friedj@mail.rockefeller.edu) or J.S.D. (; Email: dordick@ rpi.edu).

Note: Any Supplementary Information and Source Data files are available in the online version of the paper.

AUTHOR CONTRIBUTIONS

J.M.F. conceived the project, and J.M.F. and J.S.D. supervised the studies.

S.A.S. and J.S. designed and performed the experiments. R.S.K. provided technical advice. S.A.S., J.S., J.S.D. and J.M.F. wrote the manuscript.

COMPETING FINANCIAL INTERESTS

The authors declare no competing financial interests. 
has limited penetration and implanted fibers result in local activation only. Regulating gene expression in dispersed cell types has typically employed chemically mediated expression systems such as tetracycline-dependent activators or repressors ${ }^{5}$, or mutant estrogen receptor ligand-binding domains that are activated by synthetic estrogens ${ }^{6}$. However, the kinetics of drug-regulated systems are slow, taking on the order of hours to switch on gene expression and up to days to switch off as drugs are eliminated ${ }^{7}$.

Radio waves, which freely penetrate tissue, are already used clinically to control pacemakers and other devices ${ }^{8}$. Recent reports have demonstrated remote radio wave activation of engineered cells in vivo using extracellular nanoparticles ${ }^{9},{ }^{10}$ and a modified temperaturesensitive channel to control transgene expression ${ }^{9}$. However, nanoparticle injection is invasive, time limited owing to particle internalization ${ }^{11}$ and, because the applied particles only decorate cells locally, cannot be used to activate dispersed cells. Thus, a genetically encoded system for regulating gene expression and/or cellular activity in vivo provides numerous advantages. Preliminary work in vitro suggested that iron contained in the storage protein ferritin can act as an endogenous nanoparticle to activate a temperature-sensitive channel in response to $\mathrm{RF}^{9}$, but its efficacy at modulating gene expression in vivo had not been tested. Here we report the development and optimization of a robust system for remote, regulated protein production using genetically encoded nanoparticles in vitro and in vivo. We demonstrate the utility of our system using different delivery methods potentially applicable to basic and translational studies, showing that gene expression can be regulated, remotely and noninvasively, by either RFs or a magnetic field.

\section{RESULTS}

\section{In vitro optimization of RF-regulated gene expression}

We first optimized a genetically encoded system for RF-regulated gene expression by testing three separate constructs differing in the proximity of the ferritin nanoparticles to the TRPV1 channel. The first construct coexpressed a wild-type, temperature-sensitive TRPV1 cation with a cytoplasmic ferritin chimeric protein comprised of ferritin light chain, flexible linker region and ferritin heavy chain ${ }^{12}$ (TRPV1/ferritin) (Fig. 1a). The second coexpressed wild-type TRPV1 and a chimeric ferritin fusion protein, with a myristoylation signal directing ferritin to the cell membrane (TRPV1/myrferritin) (Fig. 1a). The third coexpressed a modified TRPV1 channel with an N-terminal fusion to a single-domain anti-GFP camelid antibody ${ }^{13}$, consisting of a chimeric ferritin protein with an N-terminal fusion to GFP. This results in tethering of GFP-tagged ferritin chimera to the modified TRPV1 at the cell membrane (aGFP-TRPV1/GFP-ferritin) (Fig. 1a). Immunohistochemistry for TRPV1, GFP and FLAG tag (in the flexible linker region of the ferritin chimera) in transfected HEK cells confirmed the predicted location of the expressed components (Fig. 1b). The N-terminalmodified TRPV1 was able to respond to the TRP agonist 2APB, as 2APB significantly increased intracellular calcium in HEK cells transfected with aGFP-TRPV1 (2.0-fold versus 0.85-fold change in Fluo-4 fluorescence in untransfected cells (Fig. 1c)). Finally, exposing HEK cells expressing aGFP-TRPV1/GFP-ferritin to radio waves ( $465 \mathrm{kHz}$ ) significantly increased intracellular calcium compared with what was seen in nontransfected controls (2.9-fold versus 0.8-fold change in Fluo-4 fluorescence, Fig. 1d). 
Next, we tested the efficiency of the three constructs in transducing an RF signal into gene expression in vitro using a synthetic calcium-responsive reporter gene. As previously reported ${ }^{9}$, this $\mathrm{Ca}^{++}$-sensitive promoter is comprised of a $5^{\prime}$ regulatory region of three serum response elements, three cyclic adenosine monophosphate response elements and three nuclear factor of activated $\mathrm{T}$ cell response elements upstream of a minimal promoter driving furin-modified insulin. We assayed insulin gene expression for each of the three constructs after $1 \mathrm{~h}$ of RF treatment of transfected HEK cells. TRPV1/myrferritin and aGFP-TRPV1/ GFP-ferritin all caused substantially increased calcium-dependent insulin gene expression after RF treatment (TRPV1/ferritin: RF treated $1.06 \pm 0.51$ relative insulin gene expression versus untreated $1.0 \pm 0.19$; TRPV1/myrferritin: $R F$ treated $3.16 \pm 1.17$ relative insulin gene expression versus untreated $1.0 \pm 0.19$; and aGFP-TRPV1/GFP-ferritin: RF treated 3.19 \pm 0.89 relative insulin gene expression versus untreated $1.0 \pm 0.17$, all $P<0.05)$ (Fig. 1e). The TRPV1/myrferritin and aGFP-TRPV1/GFP-ferritin showed greater induction of gene expression than TRPV1/ferritin with cytoplasmic ferritin expression. Similarly, RF treatment significantly increased proinsulin release from transfected HEK cells compared with no treatment (TRPV1/ferritin: RF treated $50.6 \pm 9.8 \mathrm{pM}$ versus untreated $17.0 \pm 0.2 \mathrm{pM}$; TRPV1/myrferritin: RF treated $63.0 \pm 4.4 \mathrm{pM}$ versus untreated $19.6 \pm 2.7 \mathrm{pM}$; and aGFPTRPV1/GFP-ferritin: RF treated $129.2 \pm 44.2 \mathrm{pM}$ versus untreated $14.8 \pm 4.3 \mathrm{pM}$, all $P<$ 0.05) (Fig. 1f). RF treatment of transfected HEK cells expressing only GFP-ferritin and insulin reporter (without TRPV1), only aGFP-TRPV1 and insulin reporter (without ferritin), or insulin reporter alone showed no significant proinsulin release (Supplementary Fig. 1). Tethering ferritin to TRPV1 using the aGFP-TRPV1/GFP-ferritin construct induced greater proinsulin release than the other two constructs. These data suggest that direct tethering of ferritin to TRPV1 is able to transduce the RF signal more efficiently than cytoplasmic or membrane ferritin expression.

\section{RF-regulated gene expression in vivo}

On the basis of these in vitro data, we tested the efficiency of the TRPV $1 /$ myrferritin and aGFP-TRPV1/GFP-ferritin constructs in vivo by implanting engineered stem cells or using a recombinant adenovirus to enable hepatic expression. Mouse mesenchymal stem cells (MSCs) were stably transfected with TRPV1/myrferritin or aGFP-TRPV1/GFP-ferritin and the calcium-dependent insulin transgene. RF treatment of MSCs expressing either construct substantially increased insulin gene expression and proinsulin release in vitro

(Supplementary Fig. 2a,b). Stably transfected MSCs grown on gelatin scaffolds ${ }^{14}$ were implanted into streptozotocin (STZ)-treated (diabetic) nude mice (Fig. 2a). TRPV1/ myrferritin and aGFP-TRPV1/GFP-ferritin-expressing MSCs were visualized on the gelatin scaffold (Fig. 2b). RF treatment of fasted mice significantly increased insulin gene expression in implanted cells expressing TRPV1/myrferritin or aGFP-TRPV1/GFP-ferritin constructs but not in control cells (Fig. 2c) (TRPV1/myrferritin: $1.8 \pm 0.3$ relative insulin gene expression versus $1.0 \pm 0.1$ basal, $P<0.05$ or aGFP-TRPV1/GFP-ferritin: $1.4 \pm 0.1$ relative insulin gene expression versus $1.0 \pm 0.1$ basal, $P<0.05$ ). RF treatment significantly increased plasma insulin in mice implanted with TRPV1/ferritin constructs (Fig. 2d) (TRPV1/myrferritin: $0.16 \pm 0.04 \mu \mathrm{IU} \mathrm{ml}^{-1}$ post-RF versus $0.07 \pm 0.01 \mu \mathrm{IU} \mathrm{ml}^{-1}$ pre-RF, $P<$ 0.05 , or aGFP-TRPV1/GFP-ferritin: $0.11 \pm 0.02 \mu \mathrm{IU} \mathrm{ml}^{-1}$ post-RF versus $0.07 \pm 0.01 \mu \mathrm{IU}$ $\mathrm{ml}^{-1}$ pre-RF, $P<0.05$ ). Blood glucose levels (Fig. 2e) fell significantly with RF treatment in 
these mice. The cumulative change in blood glucose was also decreased (area under the curve (AUC) 0-120 min) for mice implanted with TRPV1/myrferritin $(P=0.08)$ and significantly decreased for mice implanted with stem cells expressing aGFP-TRPV1/GFPferritin $(P<0.05)$ (Fig. 2f) compared with mice implanted with control cells. This shows that RF treatment of stem cells engineered to express modified TRPV1 and endogenous iron oxide nanoparticles constructs can regulate gene expression and protein release in vivo, suggesting this system could be used with engineered stem cells for regulated protein release and possibly other calcium-dependent processes in vivo. The data also suggest that tethering the ferritin particles directly to the temperature-sensitive channel improves the robustness of the system.

Next, we generated replication-deficient adenovirus with cytomegalovirus promoter-driven TRPV1/myrferritin or aGFP-TRPV1/GFP-ferritin expression followed by a stop cassette and the calcium-dependent insulin transgene (Supplementary Fig. 3a,b). We injected these adenoviruses intravenously into STZ-treated C57BL/6 mice, which resulted in hepatic expression of the constructs (Fig. 3a,b). RF treatment of fasted mice expressing either TRPV1/ferritin construct increased hepatic insulin gene expression (Fig. 3c) (TRPV1/ myrferritin: $2.3 \pm 0.4$ relative insulin gene expression versus $1.0 \pm 0.4$ basal, $P=0.08$ and aGFP-TRPV1/GFP-ferritin: $4.1 \pm 0.8$ relative insulin gene expression versus $1.0 \pm 0.1$ basal, $P<0.05$ ). Plasma insulin also increased with RF treatment in these mice but not in controls (Fig. 3d). Plasma C-peptide levels rose from undetectable levels in control mice to 3.16 \pm 1.0 pmol L ${ }^{-1}$ in mice expressing aGFP-TRPV1/GFP-ferritin (Supplementary Fig. 4). Consistent with numerous prior reports, isoflurane anesthesia increased blood glucose in control mice. The occurrence of isoflurane-induced hyperglycemia has been investigated in vitro and in vivo in dogs, rabbits and rats. In rats, isoflurane decreased glucosestimulated insulin release from isolated islets in vitro ${ }^{15}$, and in electrophysiological studies in rabbits, isoflurane reversed the inhibitory effects of glucose on pancreatic $\mathrm{K}_{\mathrm{ATP}}$ channel activity in pancreatic $\beta$ cells $^{16}$. In studies using direct portal vein sampling in dogs, isoflurane decreased insulin secretion rate and insulin pulse mass in vivo ${ }^{17}$. In both rats and rabbits, isoflurane substantially blunted glucose-stimulated insulin release in vivo ${ }^{16,18}$.

Similarly, we also found that isoflurane anesthesia caused suppression of endogenous mouse insulin release (Supplementary Fig. 5) in STZ-treated C57BL/6 mice. In contrast, RF treatment of isoflurane-treated mice expressing TRPV1/myrferritin or aGFP-TRPV1/GFPferritin reduced blood glucose (Fig. 3e,f). Intraperitoneal administration of short-acting human insulin at a dose of $0.1 \mathrm{U} \mathrm{kg}^{-1}$ of human insulin replicated the levels of insulin induced by RF and produced an equivalent reduction in blood glucose (Supplementary Fig. 6). In aggregate, these findings show that RF-regulated insulin release reduces blood glucose by replacing the insulin release blunted by isoflurane anesthesia. Further assessment with insulin clamp studies would be required to determine the effects of anesthesia and extend these findings. Additionally, expression of the apoptotic protein caspase-3 in RF-treated livers did not change (Supplementary Fig. 3c). Therefore, RF treatment of mice with viralmediated expression of TRPV1/ferritin constructs can effectively modulate gene expression and protein release in vivo. Here again the effect of the aGFP-TRPV1/GFP-ferritin construct was more robust than that of the TRPV1/myrferritin construct. Exposing fasted mice 
expressing the TRVP1/ferritin constructs to a lower RF field strength showed evidence of dose responsiveness with no significant effect of RF treatment on mice expressing TRPV1/ myrferritin and a small but significant reduction in the cumulative change in blood glucose in mice expressing aGFP-TRPV1/GFP-ferritin (Supplementary Fig. 7).

\section{Repeated RF treatment to regulate protein delivery}

To ensure the effectiveness of TRPV1 and genetically encoded nanoparticles over time, we assessed the responses of STZ-treated C57BL/6 mice expressing LacZ or aGFP-TRPV1/ GFP-ferritin and calcium-dependent insulin to weekly RF treatment. We injected mice with adenovirus expressing LacZ or aGFP-TRPV1/GFP-ferritin and calcium-dependent insulin and treated them with $1 \mathrm{~h}$ of RF once a week during weeks 2-6 after virus injection. RF treatment substantially reduced blood glucose and the cumulative changes in blood glucose (AUC 0-120 min) in aGFP-TRPV1/GFP-ferritin-expressing mice at all time points (Fig. 4a and Supplementary Fig. 8). RF also increased plasma insulin at both week 2 and week 6 (the only time points at which we bled the animals and measured plasma insulin) (Fig. $4 \mathbf{b}, \mathbf{c}$ ). Repeated RF treatment of HEK cells expressing aGFP-TRPV1/GFP-ferritin did not substantially alter expression of apoptotic markers (Supplementary Fig. 9a), and repeated RF treatment of mice did not change hepatic expression of apoptotic markers versus what was seen in untreated controls (Supplementary Fig. 9b,c).

\section{Remote activation of gene expression with a static magnetic field}

RF treatment of ferritin nanoparticles could gate TRPV1 either by particle heating and/or by delivering a mechanical stimulus to the channel through Brownian motion ${ }^{19}$. Several channels in the TRPV family have been implicated in mechanosensing ${ }^{20}$. Tethered ferritin iron oxide nanoparticles are known to have superparamagnetic properties ${ }^{21}$ and thus could transduce an external magnetic field into a mechanical force as adjacent particles align with the field ${ }^{22}$. We therefore tested whether an external magnetic field could exert a mechanical force on ferritin to activate TRPV1 channels and so regulate gene expression and protein synthesis in vitro and in vivo. We first tested this possibility in vitro using HEK cells transfected with TRPV1/ferritin, TRPV1/myrferritin or aGFP-TRPV1/GFP-ferritin.

Application of a solenoid magnetic microneedle to produce a magnetic force of $>10 \mathrm{pN}$ increased intracellular calcium in HEK cells expressing aGFP-TRPV1/GFP-ferritin with no substantial activation of TRPV1/ferritin or TRPV1/myrferritin (Supplementary Fig. 10). A standard fixed magnet (K\&J magnetics) manually moved over the cells also increased intracellular calcium in HEK cells transfected with aGFP-TRPV1/GFP-ferritin. The cumulative change in relative fluorescence $(\triangle \mathrm{F} / \mathrm{F}$ AUC $0-180 \mathrm{~s})$ for a GFP-TRPV1/GFPferritin-transfected cells was $255 \pm 8.6$ versus $159 \pm 0.6(P<0.05)$ for control cells (Fig. 5a). One-hour treatment with an intermittent magnetic field ( $5 \mathrm{~s}$ every $2 \mathrm{~min})$ substantially increased proinsulin release from HEK cells transfected with aGFP-TRPV1/GFP-ferritin and calcium-dependent insulin versus transfected cells not exposed to the magnet $(P<$ 0.001) (Fig. 5b). Magnet treatment of transfected HEK cells expressing only GFP-ferritin and insulin reporter, only aGFP-TRPV1 and insulin reporter, or insulin reporter alone did not substantially increase proinsulin release (Supplementary Fig. 1). 
We next assessed the effects of magnetic activation on gene expression in vivo in a crossover study (Fig. 5c). STZ-treated C57BL/6 mice injected with adenovirus expressing aGFPTRPV1/GFP-ferritin and calcium-dependent insulin were treated with an intermittent magnetic field (or no magnet) for $1 \mathrm{~h}$. Plasma insulin was substantially increased after magnetic treatment in mice expressing aGFP-TRPV1/GFP-ferritin and calcium-dependent insulin (Fig. 5d). Blood glucose was significantly lower in aGFP-TRPV1/GFP-ferritinexpressing mice exposed to the magnet compared to control mice during the same period ( $P$ $<0.05$ ) (Fig. 5e). Magnet treatment reduced cumulative blood glucose (AUC 0-120 min) versus untreated mice $(P<0.05)($ Fig. $5 \mathbf{f})$. This shows that RF or a magnetic field can be used to gate a ferritin-tethered TRPV1 channel in vitro and in vivo.

\section{DISCUSSION}

We report the development of a genetically encoded system for regulating gene expression in vivo using either RFs or a magnetic field. Previously, we showed that functionalized, externally delivered nanoparticles can bind epitope-tagged TRPV1 channels and transduce a remote RF signal into calcium entry and gene expression in vivo. However, this system results in regulated gene expression of local cell populations, and repeated nanoparticle administration is needed for serial responses owing to particle internalization ${ }^{11}$. A genetically encoded ferritin-tethered TRPV1 system is superior as it enables remote, robust and repeated temporal control of gene expression in vivo using either noninvasive RF fields or intermittent magnetic fields to activate TRPV1 bound to GFP-tagged ferritin that encloses iron oxide nanoparticles. We validated this genetically encoded system by showing it can regulate insulin gene expression and release in vivo to lower blood glucose. We also showed this method is effective in implanted stem cells, potentially enabling regulated $\mathrm{Ca}^{2+}$ entry and expression of key proteins in engineered stem cells. This system is highly durable as shown by longitudinal studies in adenovirus-injected mice, in which weekly RF application repeatedly regulated plasma insulin and blood glucose. Adenovirus-mediated transgene expression is limited to several weeks, so further studies examining the longevity of RFregulated gene expression will require either more durable vectors such as adeno-associated virus vector-mediated delivery or the development of transgenic mice expressing aGFPTRPV1/GFP-ferritin and a calcium-dependent reporter. These studies are currently under way.

Ferritin is a heteromultimer comprised of light and heavy chains, which creates a 5-12 nm iron oxide core ${ }^{23}$ with a complex crystalline and magnetic structure ${ }^{21}$. The iron oxide core heats in response to RF treatment ${ }^{19}$ through Néel relaxation and Brownian motion. We tested the efficiency of three ferritin locations in converting an RF field into channel activation and gene expression: cytoplasmic, membrane tethered and channel associated. We found that the GFP-tagged ferritin tethered directly to aGFP-TRPV1 robustly stimulated insulin gene expression in vitro and in vivo to a greater extent than the other constructs. Because temperature decays as the inverse distance from the particle surface, heat transfer is likely to be most efficient for this construct, suggesting that heat transfer from the particle could be limiting the efficiency of the other constructs. Alternatively, the greater efficiency of the tethered channel could suggest that the channel can be gated by mechanotransduction. We tested this possibility by exposing the ferritin-tethered TRPV1 to a magnetic field to 
exert a mechanical force without heating. The ferritin core resembles a single crystal of ferric oxyhydroxide, which is superparamagnetic with an antiferromagnetic spin arrangement ${ }^{21}$. Recent work has shown that cells overexpressing ferritin can interact with externally applied magnetic fields and that one can even pull ferritin-expressing cells off a dish using a magnet ${ }^{24}$, suggesting that tethered ferritin in a magnetic field could exert a mechanical force. Moreover, TRPV1 is a tetramer with four tethered ferritin particles that could exert a mechanical force as their orientation relative to the magnetic field either pulls them together or pushes them apart ${ }^{22}$. Our data show that ferritin tethered to TRPV1 is most effective at transducing a magnetic field into channel activation and also that TRPV1 can respond to a mechanical force. Consistent with this, we only see magnetic activation when ferritin is directly tethered to the channel and no activation even with myristoylated ferritin. This is in contrast to RF activation in which myristoylated and cytoplasmic ferritin can gate the channel albeit with lower efficiency than the tethered constructs. Together, these data raise the question of whether an oscillating magnetic field, such as RF, activates the channel by local heating or by heating and mechanical torque. The observation that a magnetic field is only capable of activating the tethered channel while RF can also activate the channel with cytoplasmic- and membrane-bound ferritin is consistent with the possibility that TRPV1 can be activated both by mechanotransduction and heat.

Systems for remote, regulated transgene expression have potential for basic research, biomanufacturing and therapeutic purposes. Defining the roles of gene products in development requires noninvasive, temporally controlled gene expression, but drug-induced gene expression using estrogens ${ }^{25}$ and tetracyclines ${ }^{26}$ can result in embryonic toxicity and have prolonged off times once the drug is removed. Both RF and magnetic fields penetrate tissue freely and may be useful in controlling transgene expression in development. Similarly, biomanufacturing processes requiring strictly regulated gene expression would benefit from remote rather than chemically induced gene expression ${ }^{27}$. Additionally, gene therapies for certain disorders require tight regulation ${ }^{28}$ or the ability to titrate transgene expression to biological response ${ }^{29}$. In this report, we have shown that ferritin tethered to TRPV1 can effectively modulate transgene expression in stem cell implants and in tissue with virally mediated transgene delivery in response to RF and magnetic fields, both of which have been used in clinical practice for setting pacemakers ${ }^{8}$ and treating depression ${ }^{30}$, respectively. Finally, because TRPV1 gates $\mathrm{Ca}^{2+}$, it is possible this system could regulate the activity of neurons as TRPV1 activation by chemical ligands has previously been shown to modulate neural activity ${ }^{31}$. If true, genetically encoded ferritin-tethered TRPV1 could be used to noninvasively activate localized or dispersed neurons or other cells in vivo using RFs or a magnetic field. Further studies, now under way, are required to determine whether ferritin-tethered TRPV1 can be used in a manner analogous to optogenetics to noninvasively regulate neural activity, potentially ushering in the use of analogous radiogenetic or magnetogenetic approaches. Additionally, the system we engineered may have similarity to endogenous magnetic sensing. Many species are capable of transducing the earth's magnetic field into a signal for navigation, and reports suggest this may be achieved through ferritinlike particles ${ }^{32}$. Knowledge of these naturally occurring systems, particularly the mechanosensing channels employed, will allow further refinement of our system ${ }^{32}$. 
In summary, we have validated the use of a genetically encoded system for noninvasive regulation of gene expression in vivo and shown that the TRPV1 channel can be activated by mechanical stimulation. These data confirm the utility of endogenously expressed nanoparticles in vivo in transducing both radio waves and magnetic fields for noninvasive control of $\mathrm{Ca}^{2+}$ entry and transgene expression.

\section{METHODS}

Methods and any associated references are available in the online version of the paper.

\section{ONLINE METHODS}

\section{Radiofrequency field}

A $465-\mathrm{kHz}$ sinusoidal signal was provided by a signal generator and applied through an amplifier (both Ultraflex, Ronkonkoma, New York) to a 2-turn solenoid coil (radius $2.5 \mathrm{~cm}$ ) to produce a field strength of 32 or $29 \mathrm{mT}$. Samples were placed within the solenoid.

\section{Application of static magnetic force}

A solenoid magnetic microneedle was fabricated by winding a 24-G copper wire around a 1/4-inch Permalloy-80 rod 1,200 times and the tip lathed to hemisphere with a diameter of $100 \mu \mathrm{m}$. Current through the coil was controlled by a Beckman $3 \mathrm{~A} / 30 \mathrm{~V}$ adjustable power supply. The needle tip was placed $\sim 30 \mu \mathrm{m}$ from the cells being pulled, and the magnetic force was produced by a 1.8-A current.

\section{Force calibration}

It has been estimated that the force to mechanically open an ion channel is approximately $0.2-0.4 \mathrm{pN}^{33}$. Therefore, we measured the force from magnetic treatment on the cells using a method described by Kim et al. Briefly, iron-loaded, ferritin-expressing cells were fixed in paraformaldehyde, added to aqueous buffer and subjected to a static magnetic field. The cells accelerate toward the magnet and their velocity is proportional to the magnitude of the magnetic force. This is modeled as a creeping flow around a sphere, where the drag of the fluid on the particle is equal to the magnetic force on the cell ${ }^{34}$. With $U$ being the cell velocity, $\mu$ being the kinematic viscosity of the buffer, and $D_{\text {cell }}$ being the cell diameter, this linear relationship is:

$$
F_{\text {drag }}=F_{\text {magnetic }}=3 \pi U \mu D_{\text {cell }}
$$

HEK cells were loaded with transferrin for $3 \mathrm{~d}$ then transfected and collected $24 \mathrm{~h}$ later. After fixation, the cells were added to buffer and subjected to magnetic field. The movement of the cells was recorded for $5 \mathrm{~s}$ in duplicate. From these two image stacks, the position of 3 cells were tracked over 10 frames for a total of $\sim 60$ cell velocities recorded. For all cells expressing ferritin, the magnetic force was over $10 \mathrm{pN}$. Cells loaded with iron but not expressing ferritin showed magnetic forces less than $1 \mathrm{pN}$ possibly owing to random Brownian motion of the fixed cells in the liquid. As $10 \mathrm{pN}$ is greater than the force required 
to open an ion channel, this experiment shows that the magnetic treatment is applying sufficient force to observe channel openings.

\section{Plasmids}

TRPV1 (in pcDNA3.1) was a kind gift from W. Liedkte (Duke University, North Carolina) and cloned into pEGFP-N1 (Clontech, Mountain View, California). A GFP-binding nanobody sequence was synthesized by Integrated DNA technologies (Coralville, Iowa) and fused to the $\mathrm{N}$ terminus of TRPV1 to create aGFP-TRPV1. A fusion protein of mouse ferritin heavy and light chains was made by inserting a synthesized flexible linker region containing a FLAG tag sequence (ARGGGGSDYKDDDDKGGGGSRV) between the ferritin chains and cloning downstream of the EF1a promoter in pCR2.1. Mouse ferritin heavy chain was obtained from ATTC (Manassas, VA) in pCMV; sport6 and mouse ferritin light chain 1 was obtained from Invitrogen (Carlsbad, CA) in pYX-Asc. pCR2.1 with EF1aferritin chimera was modified by cloning a myristoylation signal to the $\mathrm{N}$ terminus of ferritin light chain to create myrferritin or the addition of GFP sequence from Pegfp-n1 to the N terminus of ferritin light chain to create GFP-ferritin. TRPV1 followed by a 2A sequence and ferritin, myrferritin or aGFP-TRPV1 followed by a 2A sequence and GFP-ferritin were cloned into MSCV-hygro plasmids and calcium-responsive furin insulin was cloned into MSCV-puro plasmid (Clontech) for retrovirus production using Phoenix packaging cells. These sequences were also cloned into pVQ Ad CMV KNpA for generation of replicationdeficient adenovirus. The fidelity of PCR products and cloning was confirmed by DNA sequencing.

\section{Cell culture and in vitro studies}

Human embryonic kidney cells (HEK 293T (ATCC CRL-3216), mycoplasma testing and STR profiling performed by ATCC) and Phoenix ecotropic packaging cells (Stanford University) were cultured in Dulbecco's modified eagle medium with 10\% FBS (Gibco, Carlsbad, California) at $37{ }^{\circ} \mathrm{C}$ and $5 \% \mathrm{CO}_{2}$. Mouse mesenchymal stem cells (MSC) (Gibco, tested for cell-surface markers for MSC by flow cytometry and mycoplasma testing performed by Gibco) were grown in DMEM/F12 medium with $10 \% \mathrm{FBS}$ at $37{ }^{\circ} \mathrm{C}$ and $5 \%$ $\mathrm{CO}_{2}$.

Stable cell lines were produced by retroviral infection of MSCs using the Phoenix system. Briefly, Phoenix eco cells $\left(2 \times 10^{6}\right.$ cells per 6-cm dish) were transfected with MSCV-puro or hygro plasmids as above. After $24 \mathrm{~h}$, the medium was replaced and the cells placed at $32{ }^{\circ} \mathrm{C}$. Medium was aspirated after a further $24 \mathrm{~h}$, spun to remove cell debris and the supernatant added to MSCs (plated at $1 \times 10^{6}$ cells per 6-cm dish) using a 1:2 dilution in RPMI medium $/ 10 \%$ FBS with polybrene ( $4 \mu \mathrm{g} \mathrm{ml}^{-1}$, Sigma-Aldrich, St. Louis). Cells were incubated at $32{ }^{\circ} \mathrm{C}$ for a further $24 \mathrm{~h}$ and then the medium was replaced with DMEM/F12 medium $/ 10 \%$ FBS. Selection medium was added $48 \mathrm{~h}$ after infection.

Stably transfected MSC $\left(2 \times 10^{6}\right.$ cells in $60 \mu \mathrm{l}$ medium $)$ were seeded onto $5 \times 5 \times 5$-mm gelatin scaffolds (Gelfoam). Cells were maintained at $37^{\circ} \mathrm{C}$ for $4 \mathrm{~h}$, then $450 \mu \mathrm{DMEM} / \mathrm{F} 12$ medium $/ 10 \%$ FBS was added. Cell-scaffold constructs were then maintained at $37{ }^{\circ} \mathrm{C}$ for $5 \mathrm{~d}$ before implantation. 
For immunocytochemistry and RF studies, cells were cultured on 12-mm cover glass (Fisher Scientific, Pittsburgh) coated with collagen (BD Biosciences, Bedford, Massachusetts) and poly-d-lysine (Millipore, Billerica, Massachusetts). Cells were transfected with appropriate constructs $24 \mathrm{~h}$ after plating using lipofectamine 2000 (Invitrogen, Carlsbad, California). Medium was replaced $18 \mathrm{~h}$ after transfection and holotransferrin $\left(2 \mathrm{mg} \mathrm{ml}^{-1}\right.$, Sigma $)$ added to the cells. Cells were studied $72-96 \mathrm{~h}$ after transfection or subculture.

RF-dependent release of calcium-dependent human insulin-24 $\mathrm{h}$ before the study, cells were placed in $1 \%$ FBS medium at $32{ }^{\circ} \mathrm{C}$ to minimize TRPV 1 and calciumdependent pathway activation. On the day of study, cells were preincubated for $30 \mathrm{~min}$ in $500 \mu \mathrm{l}$ PBS, then incubated in $300 \mu \mathrm{l}$ of calcium imaging buffer at room temperature (control) or in an RF field at room temperature. The supernatant was removed after $60 \mathrm{~min}$, spun to remove cell debris and frozen at $-80{ }^{\circ} \mathrm{C}$ until assay. Each study was repeated on two (TRPV1/ferritin) or three occasions (TRPV1/myrferritin and aGFP-TRPV1/GFP-ferritin) each with four replicates. For gene-expression analysis, cells from the supernatant and cover glass were lysed and the lysate stored at $-80{ }^{\circ} \mathrm{C}$ until RNA purification. Each study was repeated on two (TRPV1/ferritin) or three occasions (TRPV1/myrferritin and aGFPTRPV1/GFP-ferritin) each with 4 replicates. Control studies with insulin reporter alone, aGFP-TRPV1 and insulin reporter, or GFP-ferritin and insulin reporter were performed on two occasions with four replicates. For studies of apoptosis, three groups were studied: (i) cells were placed at room temperature for $1 \mathrm{~h}$ on three occasions, (ii) cells treated with RF for $1 \mathrm{~h}$ and then placed at room temperature for a further two occasions or (iii) cells treated with RF for $1 \mathrm{~h}$ on three occasions each separated by $1 \mathrm{~h}$. At the end of the study, cells were lysed and the lysate stored at $-80{ }^{\circ} \mathrm{C}$ until RNA purification. Each study was repeated on 2 occasions each with 4 replicates.

Magnet-dependent release of calcium-dependent human insulin-Cells were prepared as described above. Cells were incubated in $300 \mu \mathrm{l}$ of calcium imaging buffer at room temperature (control) or treated with a static magnetic field for $5 \mathrm{~s}$ every $2 \mathrm{~min}$ for $1 \mathrm{~h}$ at room temperature. To produce a constant magnetic field, a neodymium-iron-boron permanent magnet was used (K\&J Magnetics, Pipersville, Pennsylvania) producing a magnetic flux density of around 5 kiloGauss near the cell surface. The supernatant was removed after $60 \mathrm{~min}$, spun to remove cell debris and frozen at $-80{ }^{\circ} \mathrm{C}$ until assay. Each study was repeated on 3 occasions, each with 4 replicates. For gene-expression analysis, cells from the supernatant and cover glass were lysed and the lysate stored at $-80{ }^{\circ} \mathrm{C}$ until RNA purification. Each study was repeated on two occasions, each with four replicates. Control studies with insulin reporter alone, aGFP-TRPV1 and insulin reporter, or GFPferritin and insulin reporter were performed on two occasions with 4 replicates. For studies of apoptosis, three groups were studied: (i) cells were placed at room temperature for $1 \mathrm{~h}$ on three occasions, (ii) cells treated with magnet for $1 \mathrm{~h}$ and then placed at room temperature for a further two occasions or (iii) cells treated with magnet for $1 \mathrm{~h}$ on three occasions each separated by $1 \mathrm{~h}$. Each study was repeated on two occasions, each with four replicates. 


\section{Calcium imaging}

Transfected cells were washed three times in PBS, then loaded with Fluo-4 $3 \mu \mathrm{M}$

(Invitrogen) in the presence of sulfinpyrazone $500 \mu \mathrm{M}$ (Sigma) or $1 \mathrm{mM}$ probenecid for 45 $60 \mathrm{~min}$ at room temperature. Cells were washed again in PBS, then incubated for 15-30 min in sulfinpyrazone in PBS. Cells were then imaged in calcium imaging buffer. Imaging was performed using a Deltavision personal DV imaging system (Applied Precision, Issawaq, Washington) equipped with a custom-made ceramic lens. Cells were imaged before and during RF, bar magnet or solenoid magnet treatment or before and after treatment with 150 $\mu \mathrm{M}$ 2-aminoethoxydiphenyl borate. Imaging was performed on at least three occasions for each condition.

The majority of cells tested with the solenoid magnetic microneedle did not respond. This is in keeping with the findings of Hughes et al. ${ }^{35}$ who noted that only a small number of cells with ion channel-tethered particles could be activated by a static magnetic field. Ion channels are sensitive to the direction of an applied force, and Hughes et al. propose that a single force direction in a static magnetic field could only open channels sensitive to that direction. Under 40× magnification, a total of approximately 2,000 cells were tested. The image stack generated for each pulling was analyzed in ImageJ. Cell fluorescence was tracked, and a $10 \%$ increase in maximum cell fluorescence over baseline $\left(F_{\max } / F_{0}\right)$ (to exclude basal calcium oscillations) was marked as a channel opening event and a responding cell.

\section{Immunocytochemistry and immunohistochemistry}

Immunocytochemistry and IHC were used to detect expression of TRPV1, GFP and FLAGtagged ferritin and to quantify apoptotic cells in cells and tissue. Cells or tissue were washed in PBS and then fixed for 15 min in 2\% paraformaldehyde (Electron Microscopy Services, Hatfield, Pennsylvania) or $10 \%$ formalin (Sigma) at $4{ }^{\circ} \mathrm{C}$ overnight then placed in $30 \%$ sucrose in PBS at $4{ }^{\circ} \mathrm{C}$ for a further $24 \mathrm{~h} .20 \mu \mathrm{m}$ cryosections were cut from tissue and placed directly on glass slides, then heated at $55^{\circ} \mathrm{C}$ for $1 \mathrm{~h}$ and stored at $-80{ }^{\circ} \mathrm{C}$ before staining. Cells or tissue sections were incubated for $1 \mathrm{~h}$ in blocking buffer (3\% BSA (Sigma) and $2 \%$ goat serum (Sigma) in PBS with $0.1 \%$ Triton-X (Sigma)). Slides were incubated in primary antibody (rabbit anti-TRPV1 1:500 (AB9554 (ref. 36), Chemicon), mouse antiFLAG 1:1,000 (Anti-FLAG M2 mouse mAb no. F1804, Sigma; ref. 37), chicken anti-GFP 1:1,000 (ab13970 (ref. 38), Abcam), rabbit anti-activated caspase-3 1:250 (G7481, Promega $\left.{ }^{39}\right)$ ) in blocking buffer overnight at $4{ }^{\circ} \mathrm{C}$. Slides were washed three times in PBS before incubation in secondary antibody (goat anti-rabbit 594 (A1012) or goat anti-rabbit 488 (A11008), goat anti-chicken 488 (A11039), goat anti-mouse 350 (A11045), all 1:1,000) in blocking buffer for $2 \mathrm{~h}$. Slides were washed a further three times in PBS before mounting using Fluoromount (Southern Biotech, Birmingham, Alabama).

Images were acquired using a Zeiss Axioplane microscope and captured digitally with separate band-pass filters using the multichannel module of the AxioVision Zeiss software. Additional images were acquired using confocal microscopy (LSM 510 laser scanning confocal microscope; Carl Zeiss MicroImaging). Quantification of active caspase-3 immunostaining was performed by an investigator blinded to the treatment group. 


\section{Animals and in vivo studies}

Male athymic $\mathrm{NCr}-\mathrm{nu} / \mathrm{nu}$ (nude) mice (NCI-Frederick, 6-8 weeks) or male C57BL/6 mice

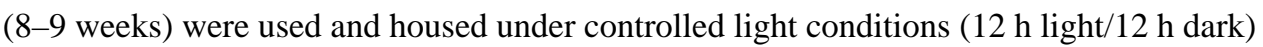
and temperature $\left(22^{\circ} \mathrm{C}\right)$, single-caged and fed ad libitum on standard mouse chow. Animal care and experimental procedures were performed with the approval of the Animal Care and Use Committee of Rockefeller University (protocol 11421) under established guidelines. In all cases, mice were randomized according to body weight after STZ treatment. The investigator was not blinded to the treatment group. The sample size required was estimated to be $n=6-8$ per group on the basis of previous studies examining the effects of RF treatment on gene expression and protein release using exogenous nanoparticles.

Study 1-Nude mice were treated for $5 \mathrm{~d}$ with low-dose streptozotocin. Two days later, MSCs seeded onto gelatin scaffolds were implanted into the flank of anesthetized nude mice bilaterally. Radiofrequency studies were performed 4 weeks later. Mice received intraperitoneal iron dextran $(50 \mu \mathrm{l}$ of $100 \mathrm{mg} / \mathrm{ml}) 5$ and $3 \mathrm{~d}$ before the study. Mice were fasted overnight before all studies. On the study day, mice with MSC implants (calciumdependent insulin alone (control), TRPV1/myrferritin with calcium-dependent insulin or aGFP-TRPV1/GFP-ferritin with calcium-dependent insulin, $n=6$-8/group) were anesthetized with inhaled isoflurane. After $30 \mathrm{~min}$, mice were treated with an RF field for 60 min. Tail vein samples were taken at $-30,0$ min before and at 15, 30, 45, 60, 75, 90 and 120 min after the onset of RF treatment. Retro-orbital blood was taken using EDTA-coated capillary tubes at -30 and $60 \mathrm{~min}$ for plasma insulin measurement. After $120 \mathrm{~min}$, approximately half the mice in each group were sacrificed and the implants removed. Each tumor was divided in two and one half snap frozen in liquid nitrogen for RNA extraction and the other half placed in 10\% formalin for IHC. Tissue was harvested from the remaining mice $24 \mathrm{~h}$ later after identical anesthesia but no RF treatment. For control group, $n=4$ no RF, 7 RF-treated; for TRPV1/myrferritin, $n=4$ no RF, 5 RF-treated; and for aGFP-TRPV1/ GFP-ferritin, $n=7$ no RF, 5 RF-treated.

Study 2-C57BL/6 mice were treated for $5 \mathrm{~d}$ with low-dose streptozotocin. Two days later, replication-deficient adenoviruses expressing LacZ, TRPV1/myrferritin with calciumdependent insulin or aGFP-TRPV1/GFP-ferritin with calcium-dependent insulin were injected into the jugular vein of anesthetized C57BL/6 mice. Iron was given as above. After 4 weeks, mice were studied as described in study 1 . For hepatic insulin gene expression, control group: $n=3$ no RF, 5 RF-treated; TRPV1/myrferritin: $n=3$ no RF, 3 RF-treated; aGFP-TRPV1/GFP-ferritin: $n=3$ no RF, 3 RF treated. For plasma insulin levels, blood glucose and cumulative change in blood glucose, control group: $n=7$, TRPV1/myrferritin: $n$ $=5$ and $\mathrm{aGFP}-\mathrm{TRPV} 1 / \mathrm{GFP}-$ ferritin: $n=6$.

Study 3-C57BL/6 mice were treated with low-dose streptozotocin and replicationdeficient adenovirus expressing LacZ, TRPV1/myrferritin with calcium-dependent insulin or aGFP-TRPV1/GFP-ferritin with calcium-dependent insulin ( $n=6 /$ group) as described above. Iron was given as above. After 3 weeks mice were treated as above. One week later, mice were treated with a lower RF field strength $(29 \mathrm{mT})$ with monitoring as above. After an additional week, mice were treated as described in study 2 but at $60 \mathrm{~min}$ after the onset of 
RF treatment, mice were sacrificed and blood taken by cardiac puncture for assessment of insulin and C-peptide. Hepatic tissue was harvested and frozen in liquid nitrogen to assess apoptotic gene expression. A set of STZ-treated, adenovirus-injected mice $(n=4-6)$ that had not received previous RF treatment were anesthetized and liver harvested and snap frozen to assess apoptotic gene expression.

Study 4-C57BL/6 mice were prepared (control, $n=7$, aGFP-TRPV1/GFP-ferritin, $n=6$ ). Mice received two doses of intraperitoneal iron dextran $(50 \mu \mathrm{lof} 100 \mathrm{mg} / \mathrm{ml}) 5$ and $3 \mathrm{~d}$ before the first study and then $3 \mathrm{~d}$ before each subsequent study. The first RF study was performed 2 weeks after virus injection and weekly thereafter until 6 weeks. The study protocol was as described for study 1 on each occasion.

Study 5-C57BL/6 mice were prepared as for study 2 . After 4 weeks, half the mice were studied using magnet stimulation using an identical protocol to study 1 but with a static magnetic field for $5 \mathrm{~s}$ every $2 \mathrm{~min}$ for $1 \mathrm{~h}$ as above and half remained untreated. One week later, the previously treated mice were assessed without magnet treatment and the previously untreated mice were treated with a static magnetic field $(n=8)$.

Study 6-C57BL/6 mice were treated with low-dose streptozotocin as described above. After 4 weeks, mice were fasted overnight, then anesthetized with inhaled isoflurane. After $15 \mathrm{~min}$, mice were treated with an intraperitoneal injection of either HumulinR $\left(0.1 \mathrm{U} \mathrm{kg}^{-1}\right)$ or an equal volume of PBS. Tail vein samples were taken at -15 and 0 min before treatment and at 15, 30, 45, 60, 90 and 120 min after injection. Retro-orbital blood was taken using EDTA-coated capillary tubes at -15 and 60 min for plasma human and mouse insulin measurement to examine the effects of isoflurane on endogenous insulin release and the effects of IP HumulinR on blood glucose and human insulin levels. PBS treated group, $n=$ 7; HumulinR treated group, $n=5$.

Assays

Proinsulin was measured in cell supernatants by ELISA (Alpco, Salem, New Hampshire) according to manufacturer's protocol. Blood glucose was determined using a Breeze 2 glucometer (Bayer, Leverkusen, Germany). Blood was spun for $10 \mathrm{~min}$ and plasma was collected. Plasma levels of human insulin were determined in mouse plasma by humanspecific ELISA (Alpco). Plasma levels of human C-peptide were determined in mouse plasma by human-specific ELISA (Mercodia, Winston-Salem, North Carolina).

\section{Real-time PCR}

Total RNA was isolated by homogenizing tissue in TRIzol reagent (Invitrogen) or cells in buffer RLT and purifying the RNA using Qiagen RNA prep kit. Complimentary DNA was synthesized using Qiagen omniscript RT kit. Real-time PCR was performed using the TaqMan system (Applied Biosystems, Foster City, California) according to the manufacturer's protocol. 


\section{Statistics}

Data over 2 s.d. outside the mean were excluded from further analysis as determined before the studies. All data were tested for Gaussian distribution and variance. Data with normal distribution and similar variance were analyzed for statistical significance using two-tailed, unpaired Student's $t$-test unless otherwise indicated. Data with normal variation and unequal variance were analyzed by two-tailed Welch's $t$-test. Paired data were analyzed by paired $t$ test. Data which were not normally distributed were analyzed by two-tailed Mann-Whitney test. $P$ values are as indicated. Data are shown as mean \pm s.e.m. unless otherwise stated.

\section{Supplementary Material}

Refer to Web version on PubMed Central for supplementary material.

\section{ACKNOWLEDGMENTS}

We would like to thank Friedman laboratory members for helpful discussions and S. Korres for assistance with preparation and submission of the manuscript. This work was funded by the Howard Hughes Medical Institute, the JPB Foundation, the US National Institutes of Health (GM095654) and a Rensselaer Fellowship (to J.S.) under a US National Institutes of Health predoctoral training grant (GM067545).

\section{References}

1. Davidson BL, Breakefield XO. Viral vectors for gene delivery to the nervous system. Nat. Rev. Neurosci. 2003; 4:353-364. [PubMed: 12728263]

2. Ando H, Furuta T, Tsien RY, Okamoto H. Photo-mediated gene activation using caged RNA/DNA in zebrafish embryos. Nat. Genet. 2001; 28:317-325. [PubMed: 11479592]

3. Cambridge SB, Davis RL, Minden JS. Drosophila mitotic domain boundaries as cell fate boundaries. Science. 1997; 277:825-828. [PubMed: 9242613]

4. Wang X, Chen X, Yang Y. Spatiotemporal control of gene expression by a light-switchable transgene system. Nat. Methods. 2012; 9:266-269. [PubMed: 22327833]

5. Gossen M, et al. Transcriptional activation by tetracyclines in mammalian cells. Science. 1995; 268:1766-1769. [PubMed: 7792603]

6. Danielian PS, Muccino D, Rowitch DH, Michael SK, McMahon AP. Modification of gene activity in mouse embryos in utero by a tamoxifen-inducible form of Cre recombinase. Curr. Biol. 1998; 8:1323-1326. [PubMed: 9843687]

7. Böcker R, Estler CJ, Maywald M, Weber D. Comparison of distribution of doxycycline in mice after oral and intravenous application measured by a high-performance liquid chromatographic method. Arzneimittelforschung. 1981; 31:2116-2117. [PubMed: 7199309]

8. Peters RW, Shafton E, Frank S, Thomas AN, Scheinman MM. Radiofrequency-triggered pacemakers: uses and limitations. A long-term study. Ann. Intern. Med. 1978; 88:17-22. [PubMed: 619753]

9. Stanley SA, et al. Radiowave heating of iron oxide nanoparticles can regulate plasma glucose in mice. Science. 2012; 336:604-608. [PubMed: 22556257]

10. Huang H, Delikanli S, Zeng H, Ferkey DM, Pralle A. Remote control of ion channels and neurons through magnetic-field heating of nanoparticles. Nat. Nanotechnol. 2010; 5:602-606. [PubMed: 20581833]

11. Knight LC, et al. Binding and internalization of iron oxide nanoparticles targeted to nuclear oncoprotein. J. Mol. Biomark. Diagn. 2010; 1:10000102. [PubMed: 23487404]

12. Iordanova B, Robison CS, Ahrens ET. Design and characterization of a chimeric ferritin with enhanced iron loading and transverse NMR relaxation rate. J. Biol. Inorg. Chem. 2010; 15:957965. [PubMed: 20401622] 
13. Kirchhofer A, et al. Modulation of protein properties in living cells using nanobodies. Nat. Struct. Mol. Biol. 2010; 17:133-138. [PubMed: 20010839]

14. Hong L, Peptan IA, Colpan A, Daw JL. Adipose tissue engineering by human adipose-derived stromal cells. Cells Tissues Organs. 2006; 183:133-140. [PubMed: 17108684]

15. Durand JL, Hosinking W, Jelicks LA. Time course of effects of inhalation anesthesia on blood glucose level in male and female C57BL/6 mice. Horm. Metab. Res. 2009; 41:339-341. [PubMed: 19148859]

16. Tanaka K, et al. Mechanisms of impaired glucose tolerance and insulin secretion during isoflurane anesthesia. Anesthesiology. 2009; 111:1044-1051. [PubMed: 19809283]

17. Vore SJ, Aycock ED, Veldhuis JD, Butler PC. Anesthesia rapidly suppresses insulin pulse mass but enhances the orderliness of insulin secretory process. Am. J. Physiol. Endocrinol. Metab. 2001; 281:E93-E99. [PubMed: 11404226]

18. Kawano T, et al. Biophysical and pharmacological properties of glucagon-like peptide-1 in rats under isoflurane anesthesia. Anesth. Analg. 2012; 115:62-69. [PubMed: 22504208]

19. Fortin JP, Gazeau F, Wilhelm C. Intracellular heating of living cells through Néel relaxation of magnetic nanoparticles. Eur. Biophys. J. 2008; 37:223-228. [PubMed: 17641885]

20. Liedtke W, et al. Vanilloid receptor-related osmotically activated channel (VR-OAC), a candidate vertebrate osmoreceptor. Cell. 2000; 103:525-535. [PubMed: 11081638]

21. Gilles C, et al. Magnetic hysteresis and superantiferromagnetism in ferritin nanoparticles. J. Magn. Magn. Mater. 2002; 241:430-440.

22. Johnsen S, Lohmann KJ. The physics and neurobiology of magnetoreception. Nat. Rev. Neurosci. 2005; 6:703-712. [PubMed: 16100517]

23. Arosio P, Ingrassia R, Cavadini P. Ferritins: a family of molecules for iron storage, antioxidation and more. Biochim. Biophys. Acta. 2009; 1790:589-599. [PubMed: 18929623]

24. Kim T, Moore D, Fussenegger M. Genetically programmed superparamagnetic behavior of mammalian cells. J. Biotechnol. 2012; 162:237-245. [PubMed: 23036923]

25. Beyer BK, Stark KL, Fantel AG, Juchau MR. Biotransformation, estrogenicity, and steroid structure as determinants of dysmorphogenic and generalized embryotoxic effects of steroidal and nonsteroidal estrogens. Toxicol. Appl. Pharmacol. 1989; 98:113-127. [PubMed: 2929019]

26. Saxén L. Drug-induced teratogenesis in vitro: inhibition of calcification by different tetracyclines. Science. 1966; 153:1384-1387. [PubMed: 4958375]

27. Fussenegger M, Schlatter S, Datwyler D, Mazur X, Bailey JE. Controlled proliferation by multigene metabolic engineering enhances the productivity of Chinese hamster ovary cells. Nat. Biotechnol. 1998; 16:468-472. [PubMed: 9592397]

28. Gadalla KK, Bailey ME, Cobb SR. MeCP2 and Rett syndrome: reversibility and potential avenues for therapy. Biochem. J. 2011; 439:1-14. [PubMed: 21916843]

29. Samaranayake H, Wirth T, Schenkwein D, Raty JK, Yla-Herttuala S. Challenges in monoclonal antibody-based therapies. Ann. Med. 2009; 41:322-331. [PubMed: 19234897]

30. Aleman A. Use of repetitive transcranial magnetic stimulation for treatment in psychiatry. Clin. Psychopharmacol. Neurosci. 2013; 11:53-59. [PubMed: 24023548]

31. Arenkiel BR, Klein ME, Davison IG, Katz LC, Ehlers MD. Genetic control of neuronal activity in mice conditionally expressing TRPV1. Nat. Methods. 2008; 5:299-302. [PubMed: 18327266]

32. Lauwers M, et al. An iron-rich organelle in the cuticular plate of avian hair cells. Curr. Biol. 2013; 23:924-929. [PubMed: 23623555]

33. St Pierre TG, Dobson J. Theoretical evaluation of cell membrane ion channel activation by applied magnetic fields. Eur. Biophys. J. 2000; 29:455-456. [PubMed: 11081406]

34. Kim T, Moore D, Fussenegger M. Genetically programmed superparamagnetic behavior of mammalian cells. J. Biotechnol. 2012; 162:237-245. [PubMed: 23036923]

35. Hughes S, McBain S, Dobson J, El Haj AJ. Selective activation of mechanosensitive ion channels using magnetic particles. J. R. Soc. Interface. 2008; 5:855-863. [PubMed: 18077244]

36. Gallego-Sandín S, Rodriguez-Garcia A, Alonso MT, Garcia-Sancho J. The endoplasmic reticulum of dorsal root ganglion neurons contains functional TRPV1 channels. J. Biol. Chem. 2009; 284:32591-32601. [PubMed: 19778904] 
37. Toriumi K, et al. SHATI/NAT8L regulates neurite outgrowth via microtubule stabilization. J. Neurosci. Res. 2013; 91:1525-1532. [PubMed: 24105954]

38. Collins CA, Kretzschmar K, Watt FM. Reprogramming adult dermis to a neonatal state through epidermal activation of $\beta$-catenin. Development. 2011; 138:5189-5199. [PubMed: 22031549]

39. Heins N, et al. Emx 2 promotes symmetric cell divisions and a multipotential fate in precursors from the cerebral cortex. Mol. Cell. Neurosci. 2001; 18:485-502. [PubMed: 11922140] 


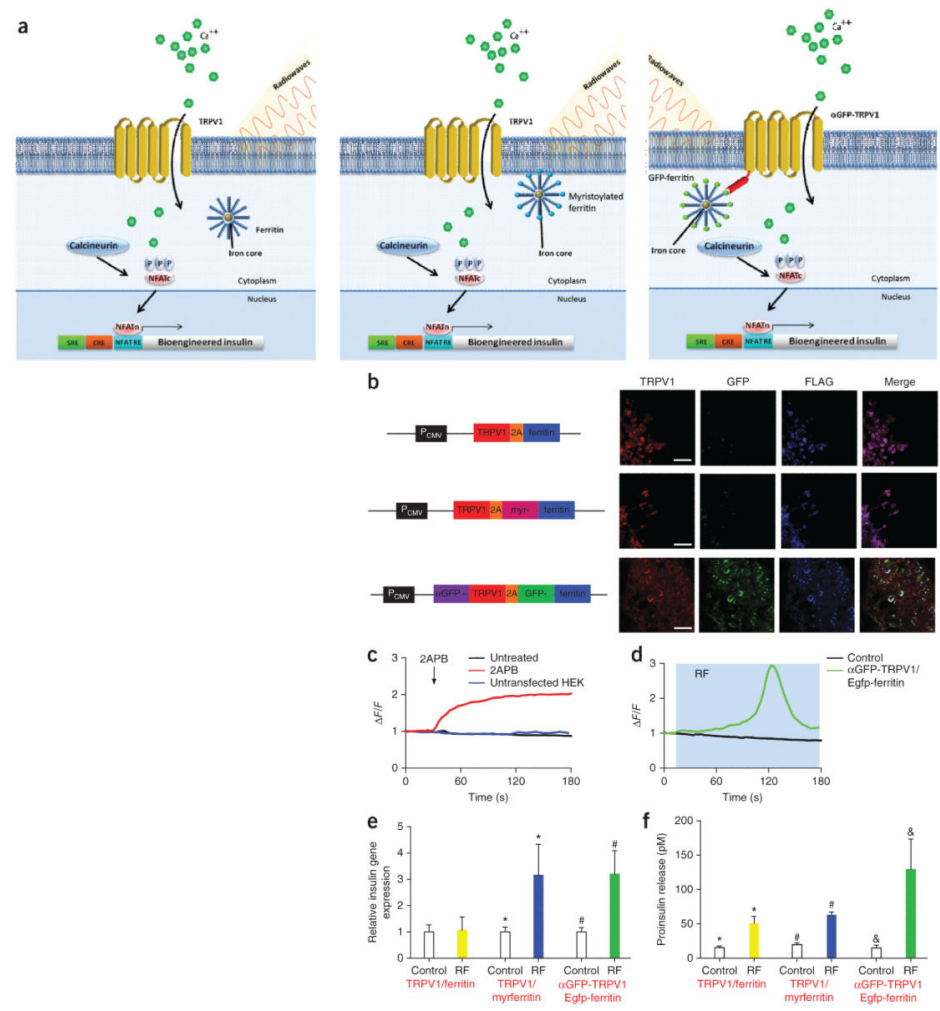

Figure 1.

In vitro optimization of gene expression and protein release with genetically encoded nanoparticles. (a) Schema of systems testing three alternate locations of genetically encoded ferritin to generate iron oxide nanoparticles to open the temperature-sensitive channel TRPV1 in response to RFs: cytoplasmic ferritin (left, TRPV1/ferritin); membrane-tethered ferritin, achieved by addition of an N-terminal myristoylation signal (middle, TRPV1/ myrferritin); and channel-associated ferritin, achieved by adding a GFP-binding domain to the $\mathrm{N}$ terminus of TRPV1 and GFP to the $\mathrm{N}$ terminus of ferritin (right, aGFP-TRPV1/GFPferritin). P, phosphate; NFATc, cytoplasmic location of nuclear factor of activated T cells; NFATn, nuclear location of nuclear factor of activated T cells; SRE, serum response element; CRE, cyclic AMP response element; NFAT RE, nuclear factor of activated T cells response element. (b) Immunohistochemistry (IHC) for TRPV1 (red), GFP (green) and FLAG-tagged (blue) ferritin chimera in HEK 293T cells transfected with TRPV1/ferritin constructs confirmed membrane expression of TRPV1 and cytoplasmic expression of ferritin (top) in cells transfected with TRPV1/myrferritin; IHC confirmed membrane expression of both TRPV1 and ferritin (middle); in cells transfected with aGFP-TRPV1/GFP-ferritin, IHC confirmed membrane expression of TRPV1, GFP and ferritin (bottom). Scale bars, $50 \mu \mathrm{m}$. (c) Representative changes in Fluo-4 fluorescence after application of the TRP agonist 2APB to untransfected HEK cells or those transfected with aGFP-TRPV1/GFP-ferritin or left untreated. (d) Representative changes in Fluo-4 fluorescence after application of RF to HEK cells transfected with aGFP-TRPV1/GFP-ferritin or without RF treatment. (e) RF treatment increases insulin gene expression in HEK cells expressing TRPV1/myrferritin and aGFP-TRPV1/GFP-ferritin but not in those expressing TRPV1/ferritin. Each study was repeated on 2 (TRPV1/ferritin) or 3 occasions (TRPV1/myrferritin and GFP-TRPV1/GFP- 
ferritin) each with 4 replicates (see Online Methods). In all cases, columns represent mean and error bars show s.e.m. Data were analyzed by a two-tailed Mann-Whitney test. * and \# indicate $P<0.05$. (f) RF treatment increases proinsulin release from HEK cells expressing TRPV1/ferritin, TRPV1/myrferritin and aGFP-TRPV1/GFP-ferritin. Each study was repeated on 2 (TRPV1/ferritin) or 3 occasions (TRPV1/myrferritin and aGFP-TRPV1/GFPferritin) each with 4 replicates. In all cases, columns represent mean and error bars indicate s.e.m. Data were analyzed by a two-tailed Mann-Whitney test. *, \# and \& indicate $P<0.05$. 

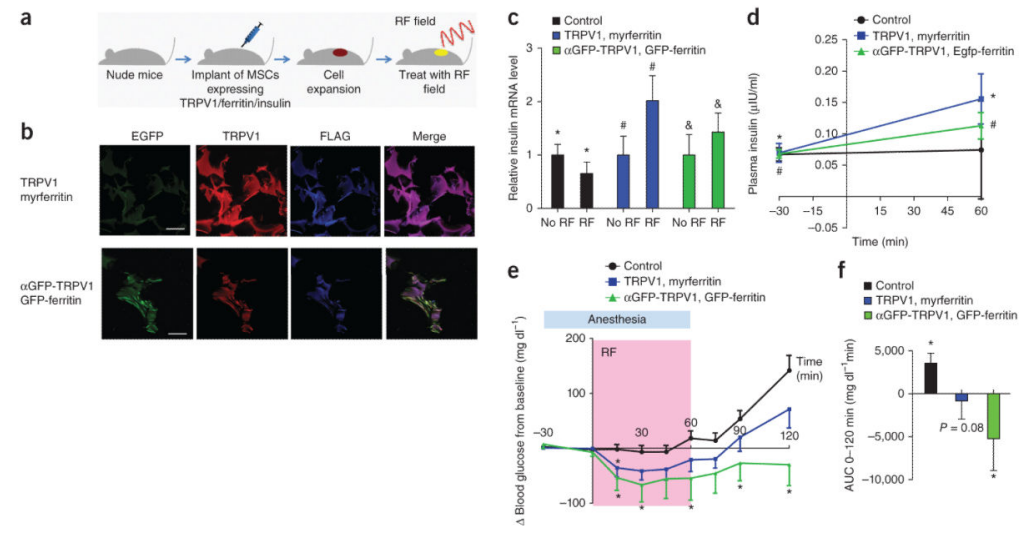

Figure 2.

RF-regulated gene expression in vivo using MSCs. (a) Schema for delivery and assessment of effects of RF treatment on blood glucose in mice with implanted MSCs expressing TRPV1/myrferritin or aGFP-TRPV1/GFP-ferritin and calcium-dependent human insulin.

(b) IHC for TRPV1, EGFP and FLAG-tagged ferritin in sections of gelatin scaffold implants seeded with MSCs stably expressing TRPV1 and myrferritin (top) or aGFP-TRPV1 and GFP-ferritin fusion (bottom). TRPV1/myrferritin-expressing cells show TRPV1 and HA staining at the cell membrane, whereas aGFP-TRPV1/GFP-ferritin-expressing cells show TRPV1, FLAG and GFP staining at the cell membrane. Scale bars, $100 \mu \mathrm{m}$. (c) Effects of RF treatment on insulin gene expression in control $(n=7)$, TRPV1/myrferritin $(n=6)$ and aGFP-TRPV1/GFP-ferritin-expressing ( $n=7)$ MSC implants. RF treatment substantially increases insulin gene expression in MSCs expressing TRPV1 and genetically encoded nanoparticles. *, \# and \& indicate $P<0.05$. Data are shown as mean \pm s.e.m. (d) Plasma insulin was substantially increased by RF treatment in mice implanted with MSCs expressing TRPV1/myrferritin ( $n=6)$ or aGFP-TRPV1/GFP-ferritin $(n=6)$ but not in control mice $(n=5)$. * and \# indicate $P<0.05$ with analysis by paired $t$-test. Error bars indicate s.e.m. (e) RF treatment of mice implanted with MSCs expressing aGFP-TRPV1/ GFP-ferritin ( $n=7)$ or TRPV1/myrferritin $(n=6)$ reduces blood glucose compared with that seen in control mice $(n=7)$. $* P<0.05$. Data are shown as mean \pm s.e.m. (f) RF treatment reduced blood glucose over the course of the study in mice implanted with MSCs expressing aGFP-TRPV1/GFP-ferritin $(n=7)$ compared with RF treatment of mice with control MSC implants $(n=7) . * P<0.05$. Data are shown as mean \pm s.e.m. 

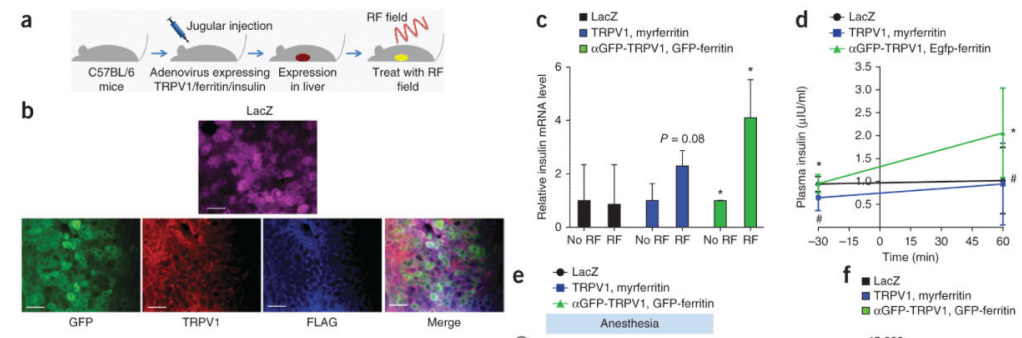

f. ${ }_{\text {Lacz }}^{\text {Time }}$

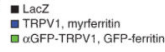

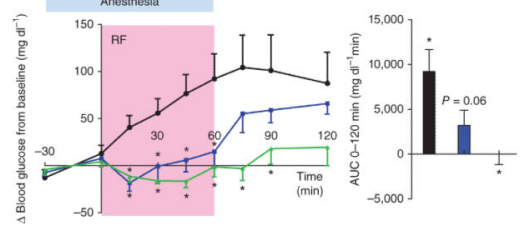

Figure 3.

RF-regulated gene expression in vivo using adenoviral delivery of transgenes. (a) Schema for delivery and assessment of effects of RF treatment on blood glucose in C57BL/6 mice injected with replication-deficient adenovirus expressing LacZ, TRPV1/myrferritin or aGFP-TRPV1/GFP-ferritin and calcium-dependent human insulin. (b) IHC for LacZ or TRPV1, EGFP and FLAG-tagged ferritin in hepatic tissue infected with adenovirus expressing LacZ (top) or with adenovirus expressing aGFP-TRPV1 and GFP-ferritin fusion (bottom). Scale bars, $100 \mu \mathrm{m}$. (c) Effects of RF treatment on hepatic insulin gene expression in mice treated with adenovirus expressing LacZ, TRPV1/myrferritin or aGFP-TRPV1/ GFP-ferritin and calcium-dependent human insulin. For hepatic insulin gene expression, control group: $n=3$ no RF, 5 RF-treated; TRPV1/myrferritin: $n=3$ no RF, 3 RF-treated; aGFP-TRPV1/GFP-ferritin: $n=3$ no RF, 3 RF treated. RF treatment substantially increases insulin gene expression in hepatic tissue expressing aGFP-TRPV1/GFP-ferritin. ${ }^{*} P<0.05$. Error bars indicate s.e.m. (d) Plasma insulin was increased by RF treatment in mice expressing TRPV1/myrferritin ( $n=5)$ or aGFP-TRPV1/GFP-ferritin $(n=6)$ but not in control mice $(n=7){ }^{*}$ and \# indicate $P<0.05$ with analysis by paired $t$-test. Data shown as mean \pm s.e.m. (e) RF treatment of mice injected with adenovirus expressing aGFP-TRPV1/ GFP-ferritin $(n=6)$ reduced blood glucose compared with that seen in control mice $(n=7)$. Asterisks indicated $P<0.05$; data shown as mean \pm s.e.m. (f) RF treatment substantially reduced blood glucose over the course of the study in mice expressing aGFP-TRPV1/GFPferritin $(n=6)$ compared with RF treatment of mice expressing LacZ $(n=7) . * P<0.05$. Data are shown as mean \pm s.e.m. 

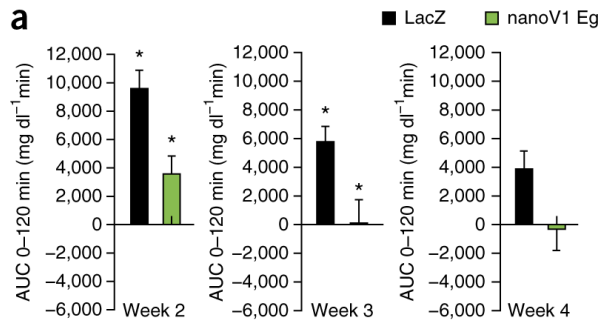

- LacZ $\square$ nanoV1 Egfp-ferritin
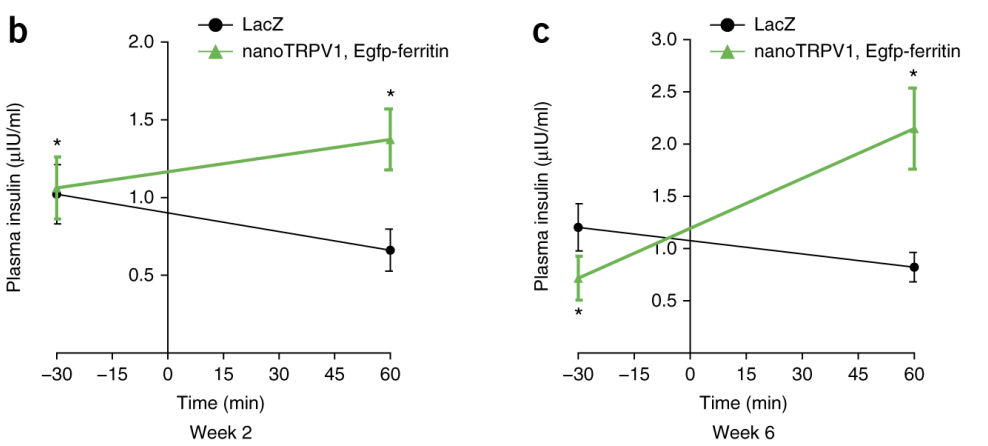

Figure 4.

Repeated RF treatment to regulate protein delivery. (a) Effects of RF treatment at weeks 2-6 after virus injection on cumulative blood glucose in C57BL/6 mice injected with control ( $n=$ 7) or aGFP-TRPV1/GFP-ferritin $(n=6)$ expressing adenovirus. RF treatment reduced cumulative blood glucose in aGFP-TRPV1/GFP-ferritin-expressing mice at each assessment. $* P<0.05$. Data are shown as mean \pm s.e.m. (b) Plasma insulin was increased by RF treatment in mice expressing aGFP-TRPV1/GFP-ferritin $(n=6)$ but not in control mice $(n=7)$ at week 2. $* P<0.05$. Error bars indicate s.e.m. (c) Plasma insulin was increased by $\mathrm{RF}$ treatment in mice expressing aGFP-TRPV1/GFP-ferritin $(n=6)$ but not in control mice $(n=6)$ at week $6 . * P<0.05$ with analysis by paired $t$-test. Data are shown as mean \pm s.e.m. 


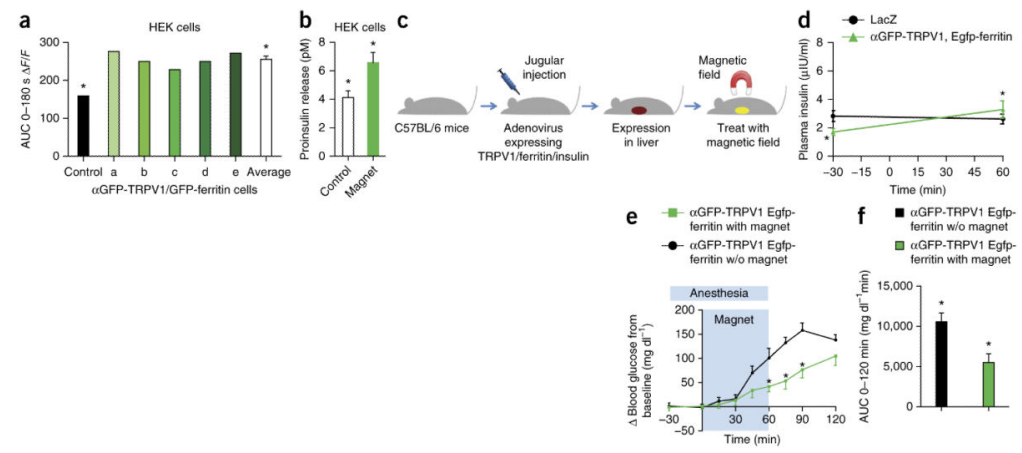

Figure 5.

Remote activation of gene expression with a static magnetic field. (a) Effects of magnetic field on cumulative changes in Fluo-4 fluorescence in five representative HEK cells transfected with aGFP-TRPV1/GFP-ferritin or control cells. $* P<0.05$. Data are shown as mean \pm s.e.m. $n=6$. (b) Magnetic field treatment increases proinsulin release from HEK cells expressing aGFP-TRPV1/GFP-ferritin and calcium-dependent human insulin. Data are shown as mean \pm s.e.m. Each study was repeated on 3 occasions, each with 4 replicates. $* P$ $<0.05$ as analyzed by Mann-Whitney test. (c) Schema for delivery and assessment of effects of magnet treatment on blood glucose in C57BL/6 mice injected with replication-deficient adenovirus expressing aGFP-TRPV1/GFP-ferritin and calcium-dependent human insulin. (d) Plasma insulin is substantially increased in mice expressing aGFP-TRPV1/GFP-ferritin and calcium-dependent human insulin treated with an intermittent magnetic field compared with no magnet treatment. ${ }^{*} P<0.05$ as analyzed by paired $t$-test. (e) Magnet treatment reduced blood glucose over the course of the study in mice expressing aGFP-TRPV1/GFPferritin compared with no magnet treatment. $* P<0.05$. Data are shown as mean \pm s.e.m. (f) Magnet treatment reduced blood glucose over the course of the study in mice expressing aGFP-TRPV1/GFP-ferritin compared with magnet treatment of mice expressing LacZ. $* P<$ 0.05 . Data are shown as mean \pm s.e.m. 